and Chemical Societies, the Reviews of Modern Physics, Chemical Reviews and Die Physik. With the persistence of the Government restrictions placed upon science, German periodicals will become less and less important. To avoid duplication it is therefore necessary to secure co-operation between only English-speaking societies.

The Physical Society is already broadminded enough to adopt in its Proceedings an indexing system designed to help in the international co- ordination of knowledge. Is it too much to hope that the Society will also try to secure the co-operation necessary to present an annual report holding a unique position in journals published in English ? Even the present "Reports" are indispensable in any science library, and readers are heavily indebted to the many individual contributors who have had to sacrifice some of their research effort to carry out the arduous task of digesting hundreds of research papers.
W. H. George.

\title{
Light in the Service of Man
}

\section{Allgemeine Photochemie:}

ein Hand- und Lehrbuch für Studium und Forschung für Mediziner, Biologen, Agrikulturchemiker, Botaniker, usw. Von Prof. Dr. J. Plotnikow. Zweite, umgearbeitete und erweiterte Auflage. Pp. viii +909. (Berlin und Leipzig: Walter de Gruyter und Co., 1936.) 28.50 gold marks.

DROF. J. PLOTNIKOW, who has considerable experience in theoretical and applied photochemical research, has dealt with different aspects of photochemistry satisfactorily in this treatise under notice. He has divided the subject-matter into four sections, namely : (1) sun and mankind ; (2) interaction of matter and radiation energy; (3) statics and dynamics of light reactions; (4) different reactions caused by light.

In the first section, the characteristic properties of different radiations (for example, infra-red, visible, ultra-violet, etc.) and the problem of sight are described in detail. In the same section, the subject of nutrition in the future has been considered in an interesting manner from the point of view of the photochemist. A brief historical account of the development of photochemistry from ancient times appears at the end of this part.

In the second section, the laws of light absorption, reflection and scattering, the phenomena of fluorescence, phosphorescence, bioluminescence, etc., the laws and mechanism of photochemical reactions, temperature coefficient, quantum yield and allied problems, are discussed. It is interesting to note that the summary of quantum yields of different photochemical reactions recorded on pages 230-231 shows that the Einstein law of photochemical equivalence fails in the majority of reactions investigated so far. This fact has also been emphasized by the reviewer.

The mathematical aspect of photochemistry is considered in the third part of the book. The experimental technique of photochemical reactions, which has been considerably developed by Prof. Plotnikow and co-workers, is also discussed in this section. Cases of photochemical catalysis and equilibrium and the relation between the intensity of the incident radiation and the velocity of photochemical reactions have also been included.

In the fourth part, the author describes briefly numerous photochemical reactions involving inorganic and organic compounds. Most of the literature in photochemistry has been condensed in this section, which also contains a good account of the mechanism of photosynthesis in plants and the function of chlorophyll. Photolysis and photo-oxidations are also adequately considered. At the end, work on the sensitization of photographic plates and films to various parts of the spectrum is summarized: to the infra-red radiation by neocyanine, kryptocyanine, xenocyanine, etc. ; to red by dicyanine ; to orange red by pinacyanol, nigrosin, chlorophyll, etc. ; to orange by pinachrome, cyanine, orthochrome-T, methyl violet, ethyl violet, etc. ; to blue green by uranine, acridine yellow, etc.; to ultraviolet by sodium salicylate (alcoholic solution), 'Vaseline' (acetone solution) and other fluorescent substances. A brief account of the phenomenon of desensitization of photographic plates and films with phenosafranine and allied compounds dis. covered by Lüppo-Cramer has also been included.

As light is utilized in the production of food materials in plants, in nitrogen fixation and in other processes in the soil, in photography, in the production of vitamin, in the treatment of disease and in the preservation of health, in the large-scale sterilization of water and milk, in the detection and testing of different materials, etc., photochemistry is of necessity engaging the attention of numerous workers all over the world, and several important books on this topic have recently been published. The present comprehensive work by Prof. Plotnikow is a valuable contribution to knowledge and should be widely read by chemists, physicists, biologists, agriculturists, medical men and others interested in this subject, which has great possibilities. 